\title{
Discovery of very high energy gamma-ray emission from 1FGL J2001.1+4351 by MAGIC
}

\author{
Karsten Berger ${ }^{1}$ \\ Instituto de Astrofisica de Canarias \\ Vía Láctea, E38205 - La Laguna (Tenerife), Spain \\ Universidad de La Laguna \\ Molinos de Agua, E38207 - La Laguna (Tenerife), Spain \\ E-mail: bergereiac.es

\section{David Paneque} \\ Max-Planck-Institut für Physik (Werner-Heisenberg-Institut) \\ Föhringer Ring 6, D80805 - München, Germany \\ SLAC National Accelerator Laboratory, Stanford University \\ CA 94305 - Stanford,USA \\ E-mail: dpaneque emppmu.mpg.de
}

\section{Gianluca Giavitto}

Institut de Física d'Altes Energies (IFAE)

Edifici Cn, Universitat Autónoma de Barcelona (UAB), E-08193 - Bellaterra (Barcelona), Spain

E-mail: gttolifae.es

\begin{abstract}
We report the discovery of Very High Energy (VHE; >100 GeV) gamma-ray emission from the source 1FGL J2001.1+4351, (RA 2001 13.5, dec 4353 02.8, J2000), which is positionally consistent with the location of the flat spectrum radio source MG4 J200112+4352 (RA 2001 12.9, dec 435252.8 , J2000). The VHE detection is based on a 1.5 hour-long observation performed on July 16 th in stereoscopic mode with the two $17 \mathrm{~m}$ diameter imaging Cherenkov telescopes on La Palma, Canary Islands, Spain. The preliminary analysis of the MAGIC data using the standard cuts optimized for soft energy spectra sources yields a detection of 125 gamma-rays above $90 \mathrm{GeV}$, corresponding to a pre-trail statistical significance of 7.6 standard deviations. The observed flux is estimated to be $\sim 20 \%$ of the Crab nebula flux above $100 \mathrm{GeV}$. Earlier MAGIC observations indicated a substantially lower flux; hence indicating that the source is variable on a few days timescale.
\end{abstract}

5th Texas Symposium on Relativistic Astrophysics -TEXAS2010

Heidelberg, Germany

December $06-112010$

For the MAGIC Collaboration 


\section{Introduction}

The high-energy gamma-ray sky is very diverse. The "Fermi Large Area Telescope First Source Catalogue" (hereafter 1FGL) lists the sources detected at energies above $0.1 \mathrm{GeV}$ in the first 11 months of science operations [1]. The list comprises 820 sources that have been identified or associated, and 631 sources that have not been associated with potential counterparts at other wavelengths. The 1FGL catalogue provides the scientific community with an unprecedented description of the gamma-ray sky, which will be very helpful for both individual source and population studies. Since the spectral energy distribution of many (if not most) of the Large Area Telescope (LAT) sources covers several decades in energy, multifrequency observations are essential in order to fully characterize their spectra and understand the physical processes occurring in those sources.

The most sensitive instruments to observe at Very High Energy (VHE; >100 GeV) are the Imaging Atmospheric Cherenkov Telescopes (IACTs), which detect gamma-rays through the detection of the Extended Air Showers (EAS) induced by the primary gamma-ray in the Earth atmosphere. In this case, the atmosphere serves as a calorimeter, and large effective collection areas of $10^{4-5} \mathrm{~m}^{2}$ can be achieved. This detection efficiency needs to be compared with that of the Fermi-LAT which is about $1 \mathrm{~m}^{2}$. The most advanced IACTs are currently HESS [2], VERITAS [3] (arrays of four $\sim 12 \mathrm{~m}$ diameter mirror telescopes) and MAGIC [4], [5] (stereoscopic array consisting of two $\sim 17 \mathrm{~m}$ diameter telescopes). Among all of them, MAGIC is the IACT with the lowest trigger energy threshold $(60 \mathrm{GeV})$, and hence the one that has the largest overlap with the energy range covered by the Fermi-LAT (nominally $20 \mathrm{MeV}-300 \mathrm{GeV}$ ). In these proceedings we report the discovery of VHE gamma-ray emission from the 1FGL J2001.1+4351, which was observed by MAGIC during summer 2010.

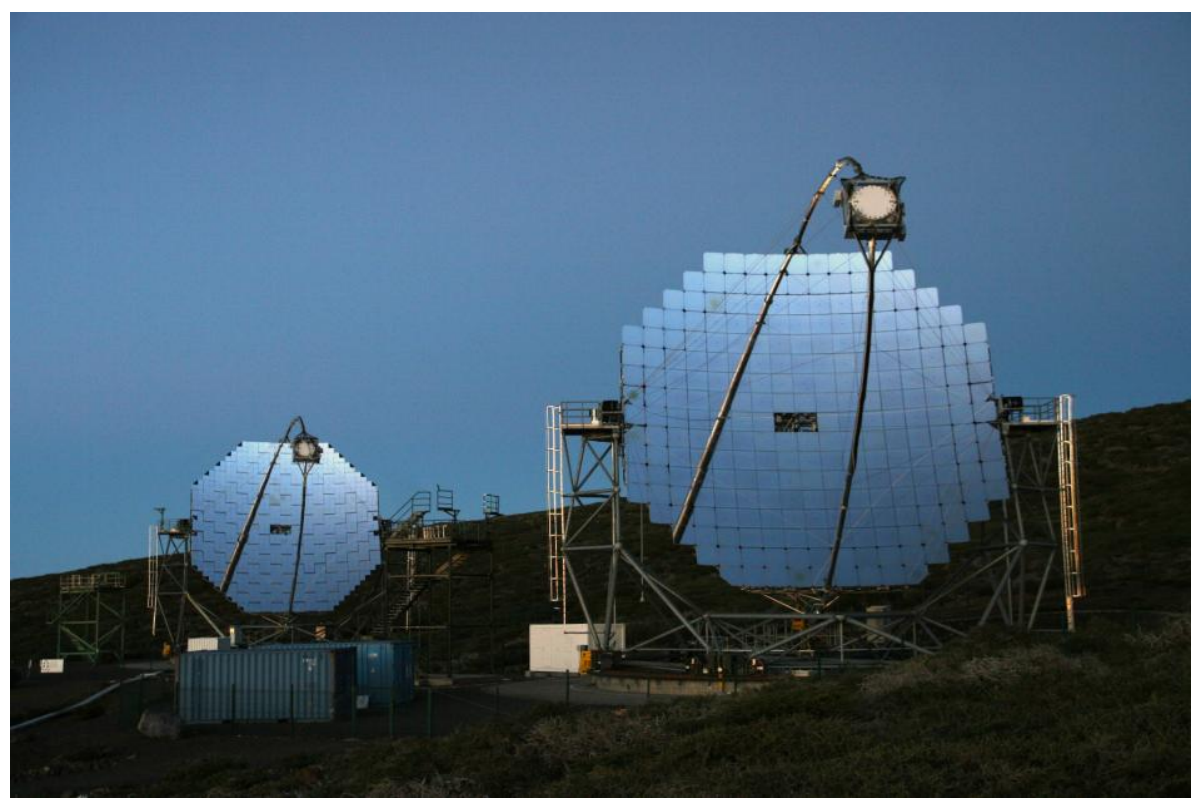

Figure 1: The MAGIC 17m imaging atmospheric Cherenkov telescopes at the Roque de los Muchachos Observatory, La Palma, Spain. Both telescopes are $85 \mathrm{~m}$ apart. Image copyright: Robert Wagner. 


\section{The unidentified Fermi source 1FGL J2001.1+4351}

Remarkably 1FGL J2001.1+4351 was first detected by the Fermi-LAT only above $1 \mathrm{GeV}$ [1]. The unknown identification of the object triggered a study by Bassani et al. [6] in the optical, radio and X-ray band. The most likely counterpart, MG4 J200112+4352 (a bright, flat spectrum radio source), was subsequently classified as a high frequency peaked BL Lacertae object and thus an active galactic nucleus, whose jet is pointing directly towards our line of sight (a so-called "blazar"). The source was found to be variable both in the X-ray as well as the optical band. The redshift of this source is still unknown, though the identification of the optical host galaxy suggests $\mathrm{z}<0.2$ [6]. This source was flagged as a very promising VHE source candidate (together with other sources), based on a dedicated search and spectral analysis above 10 and $30 \mathrm{GeV}$ in the Fermi-LAT data accumulated over 1 year. A similar analysis using the accumulated data over 2+ years was reported in this conference [7]. The Fermi-LAT collaboration shared this information (list of VHE candidate sources based on 1 year data) with the MAGIC collaboration (as well as other IACTs) back in October 2009, and motivated the observations of this source with MAGIC in summer 2010.

\section{MAGIC Discovery and Outlook}

MAGIC observed 1FGL J2001.1+4351 during July until September 2010. On July $16^{\text {th }}$ during a $1.5 \mathrm{~h}$ long, observation a clear excess was found at the position of 1FGL J2001.1+4351. The preliminary analysis of the MAGIC data using the standard cuts optimized for soft energy spectra sources yields a detection of 125 gamma-rays above $90 \mathrm{GeV}$, corresponding to a pretrail statistical significance of 7.6 standard deviations [8]. The observed flux is estimated to be $\sim 20 \%$ of the Crab nebula flux above $100 \mathrm{GeV}$. Earlier MAGIC observations indicated a substantially lower flux; hence indicating that the source is variable on a few days timescale. Observations with Swift have also been performed on this source location. A preliminary analysis of the Swift/XRT data shows that the X-ray flux from July 16th 2010 is $\sim 3$ times larger than the X-ray flux measured during previous Swift observations in July 2010, hence indicating that the source is variable (on few days timescale) and that it was X-ray active during the MAGIC observations on July 16th, indicating a correlation between VHE and X-ray activity at that time.

Multifrequency observations were performed before, during and after the MAGIC VHE detection with various instruments covering energies from radio (e.g. OVRO), optical (e.g. KVA, GRT, Galaxy View observatory), UV (Swift/UVOT) and X-rays (Swift/XRT and RXTE/PCA). In addition, the gamma-rays from Fermi-LAT were continuously collected during all these observations. The multifrequency variability, correlations and scientific implications of the results will be reported in a forthcoming publication, where we will also address the possibility of intra-night variability in the VHE data. 


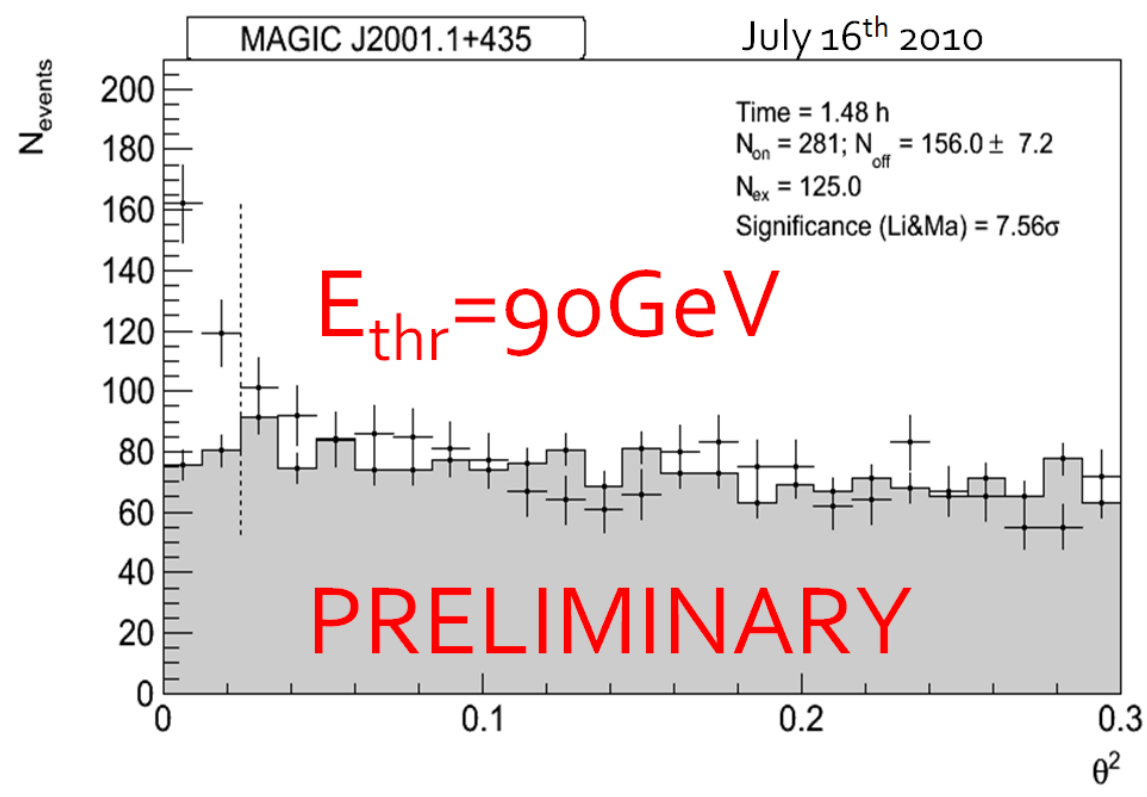

Figure 2: Squared angular distribution with respect to the position of 1FGL J2001.1+4351. Observation details are given in the inlay. As expected for the detection of a VHE $\gamma$-ray source the observed excess is peaked towards the position of $\mathbf{J 2 0 0 1 . 1 + 4 3 5 1}$. The energy threshold of this preliminary analysis is $\sim 90 \mathrm{GeV}$. 


\section{References}

[1] A. A. Abdo et al. (Fermi Coll.), FERMI LARGE AREA TELESCOPE FIRST SOURCE CATALOG, ApJS 188 (2010) 405

[2] Hinton J. A. (H.E.S.S. Coll.), The status of the H.E.S.S. project, New Astron. Rev., 48 (2004) 331

[3] Weekes T. C. et al., Astropart. Phys., 17 (2002) 221

[4] Lorentz, E. et al., Status of the 17m diameter MAGIC telescope, New Astron. Rev., 48 (2004) 339

[5] K. Berger et al. (MAGIC Coll.), Recent results of extra-galactic observations with the MAGIC telescope system, PoS(Texas 2010) 176.

[6] L. Bassani et al., On the identification of the Fermi/LAT source OFGL J2001.0+4352 with a BL Lac, MNRAS 397 (2009) L55.

[7] Paneque and Digel (Fermi Coll.), PoS(Texas 2010).

[8] M. Mariotti et al. (MAGIC Coll.), Discovery of Very High Energy gamma-ray emission from 1FGL J2001.1+4351 by MAGIC, Atel \#2753 